\title{
Simultaneous analysis of several models in the three-dimensional Ising universality class
}

\author{
Youjin Deng ${ }^{1}$ and Henk W.J. Blöte ${ }^{1,2}$ \\ ${ }^{1}$ Faculty of Applied Sciences, Delft University of Technology, P.O. Box 5046, 2600 GA Delft, The Netherlands \\ ${ }^{2}$ Lorentz, Institute, Leiden University, P.O. Box 9506, 2300 RA Leiden, The Netherlands
}

(Received 25 April 2003; published 23 September 2003)

\begin{abstract}
We investigate several three-dimensional lattice models believed to be in the Ising universality class by means of Monte Carlo methods and finite-size scaling. These models include spin- $\frac{1}{2}$ models with nearestneighbor interactions on the simple-cubic and on the diamond lattice. For the simple cubic lattice, we also include models with third-neighbor interactions of varying strength, and some "equivalent-neighbor" models. Also included are a spin-1 model and a hard-core lattice gas. Separate analyses of the numerical data confirm the Ising-like critical behavior of these systems. On this basis, we analyze all these data simultaneously such that the universal parameters occur only once. This leads to an improved accuracy. The thermal, magnetic, and irrelevant exponents are determined as $y_{t}=1.5868(3), y_{h}=2.4816(1)$, and $y_{i}=-0.821(5)$, respectively. The Binder ratio is estimated as $Q=\left\langle m^{2}\right\rangle^{2} /\left\langle m^{4}\right\rangle=0.62341(4)$.
\end{abstract}

DOI: 10.1103/PhysRevE.68.036125

PACS number(s): 05.50.+q, 64.60.Cn, 64.60.Fr, 75.10.Hk

\section{INTRODUCTION}

The Ising model has been investigated extensively, and thus serves as a testing ground for theories of phase transitions. Many physical systems can be described by this simple but nontrivial model. It is believed that continuous phase transitions in systems with short-range interactions and a scalar order parameter belong to the Ising universality class. These include a variety of magnetic systems, alloys, gasliquid systems, and liquid mixtures. For instance, magnetic systems can be described by a spin- $\frac{1}{2}$ or spin- 1 Ising model depending on the nature of the elementary magnetic moments; gas-liquid systems can be modeled by means of hardcore particles, which exclude one another within a nonzero range. Furthermore, the particle coordinates may be restricted to the vertices of regular lattices.

In two dimensions, the evidence supporting the universality hypothesis is rather solid. One underlying reason is that exact results are available. For instance, exact analysis of Onsager's spin- $\frac{1}{2}$ model [1] and related models yields the thermal and magnetic scaling exponents as $y_{t}=1$ and $y_{h}$ $=15 / 8$ [2], respectively. In three dimensions, however, such exact results are absent. Therefore, investigation of critical behavior has to depend on approximations. These include techniques such as $\epsilon$ and series expansions, the coherentanomaly method, and Monte Carlo methods, etc. Extensive studies have been carried out [3-14], and there is some consensus that the values of $y_{t}$ and $y_{h}$ are, respectively, 1.587 and 2.482, with differences only in the last decimal place. Compared to the case of two dimensions, the threedimensional results are indeed less satisfactory. Apart from the limited accuracy, the absence of exact results leaves, at least in principle, some room for severe disagreements. For instance, a very recent investigation by García and coworkers [15] claims that $y_{t}=1.600(2)$ and $y_{h}=2.501(5)$.

Many factors are responsible for this unsatisfactory situation. First, due to the restriction of current computer capacity, one can only explore rather limited system sizes in three dimensions. Second, corrections to scaling are much more serious than that in two dimensions. For the two-dimensional
Ising model, the exponent of the leading irrelevant thermal field is $y_{i}=-2$, while in three dimensions $y_{i} \simeq-0.82$. Moreover, the determination of $y_{i}$ is not very accurate so far. A better estimation of $y_{i}$ thus seems justified and is one of the purposes of the present paper.

In the language of renormalization group technique, the critical behavior of systems within a universality class is governed by a common fixed point. In terms of scaling fields, the function of the free energy, and thus of physical observables, is universal near the critical points. By means of finite-size scaling, such universal functions are extended to finite systems. As an example, we concern the dimensionless ratio $Q=\left\langle m^{2}\right\rangle^{2} /\left\langle m^{4}\right\rangle$, where $m$ is the profile of order parameter. The quantity $Q$ is related to Binder cumulant [16], and has been reported [11] to be a good choice to estimate $y_{i}$ and locate criticality. Near the critical points $Q$ behaves as

$$
Q(t, v, L)=Q\left(t L^{y_{t}}, v L^{y_{i}}, 1\right)+\cdots,
$$

where $L$ is the linear system size, $t$ is the thermal scaling field, and the irrelevant field $v$ reflects the distance of criticality of corresponding systems and the fixed point. Here, we have not yet specified contributions due to the analytic part of the free energy. Taylor expansion of the right-hand side of Eq. (1) yields

$$
\begin{aligned}
Q(t, v, L)= & Q^{(0)}+Q^{(1,0)} t L^{y_{t}}+Q^{(2,0)} t^{2} L^{2 y_{t}}+Q^{(0,1)} v L^{y_{i}} \\
& +\cdots,
\end{aligned}
$$

where the derivatives of the universal function $Q$ with respect to $t$ and $v$ are denoted as $Q^{(i, j)}$. Apart from the scaling exponents $y_{t}$ and $y_{i}$, the amplitudes of $Q^{(0)}$ and $Q^{(i, j)}$ are equal for systems in the same universality class.

However, from the Monte Carlo data of a single model only, the estimation of $y_{i}$ is rather difficult. The reason is as follows. In Eq. (2), the amplitude $v$ is coupled to the exponent $y_{i}$, and thus a reasonable estimation of $y_{i}$ requires systems with a large value of $v$. However, the large value of $v$ excludes an accurate determination of $Q^{(0)}$, so that the accuracy of $y_{i}$ is also limited. On the other hand, although a 
TABLE I. Definitions of the models.

\begin{tabular}{|c|c|c|c|c|c|c|}
\hline Model & $\frac{K_{2 \mathrm{~N}}}{K_{\mathrm{NN}}}$ & $\frac{K_{3 \mathrm{~N}}}{K_{\mathrm{NN}}}$ & $\frac{K_{4 \mathrm{~N}}}{K_{\mathrm{NN}}}$ & $D$ & Lattice & Description of models \\
\hline 1 & 0 & 0 & 0 & $-\infty$ & $\mathrm{d}$ & Spin- $\frac{1}{2}$ with NN couplings \\
\hline 2 & 0 & 0 & 0 & $-\infty$ & $\mathrm{sc}$ & Spin- $\frac{1}{2}$ with NN couplings \\
\hline 3 & 0 & 0.1 & 0 & $-\infty$ & $\mathrm{sc}$ & Spin- $\frac{1}{2}$ with NN and $3 \mathrm{~N}$ couplings \\
\hline 4 & 0 & 0.2 & 0 & $-\infty$ & $\mathrm{sc}$ & Spin- $\frac{1}{2}$ with $\mathrm{NN}$ and $3 \mathrm{~N}$ couplings \\
\hline 5 & 0 & 0.3 & 0 & $-\infty$ & $\mathrm{sc}$ & Spin- $\frac{1}{2}$ with $\mathrm{NN}$ and $3 \mathrm{~N}$ couplings \\
\hline 6 & 0 & 0.4 & 0 & $-\infty$ & $\mathrm{sc}$ & Spin- $\frac{1}{2}$ with $\mathrm{NN}$ and $3 \mathrm{~N}$ couplings \\
\hline 7 & 1 & 0 & 0 & $-\infty$ & $\mathrm{sc}$ & Equivalent neighbor of order 2 \\
\hline 8 & 1 & 1 & 0 & $-\infty$ & $\mathrm{sc}$ & Equivalent neighbor of order 3 \\
\hline 9 & 1 & 1 & 1 & $-\infty$ & $\mathrm{sc}$ & Equivalent neighbor of order 4 \\
\hline 10 & 0 & 0 & 0 & $\ln 2$ & $\mathrm{sc}$ & Spin-1 with NN couplings \\
\hline 11 & & & & & $\mathrm{sc}$ & Lattice gas with NN exclusion \\
\hline
\end{tabular}

${ }^{a} \mathrm{~d}$ diamond lattice; sc simple-cubic lattice.

system with a small amplitude $v$ helps to estimate $Q^{(0)}$, it does not allow a good determination of $y_{i}$ either. This is one of the reasons why, in many numerical investigations, the exponent $y_{i}$ is fixed at a constant taken from other sources.

This problem can be avoided by a simultaneous analysis of several systems with a diversity of the irrelevant fields $v$. Given a reasonable value of $y_{i}$, Monte Carlo data of systems with a small irrelevant field $v$ determine $Q^{(0)}$ with a narrow margin; this information, together with models with a significant amplitude $v$, greatly helps the estimation of $y_{i}$, which in return improves the determination of $Q^{(0)}$.

In the present paper, we investigate 11 Ising-like lattice models in three dimensions, of which the amplitudes $v$ have a wide range of values. These models include the spin $-\frac{1}{2}$ Ising model with nearest-neighbor interactions $K_{\mathrm{NN}}$ on the simple cubic and on the diamond lattice. On the simple-cubic lattice, models with further-neighbor interactions are also investigated. In particular, third-neighbor interactions $K_{3 \mathrm{~N}}$ are included in several models with various ratios $K_{3 \mathrm{~N}} / K_{\mathrm{NN}}$. Further, we study some "equivalent-neighbor" models, originally introduced by Domb and Dalton [17-19]. In such systems, each spin interacts equally strongly with all its neighbors within a certain distance. The model with the interactions till the $r$ th shell of neighbors is referred to as the equivalent-neighbor model of the order of $r$. Also included are a spin-1 model and a hard-core lattice gas with nearestneighbor exclusion $[20,21]$.

For these models, we analyze the numerical data both separately and simultaneously. The separate analyses are in a good agreement with the Ising universality hypothesis for all these systems. This provides the basis of the simultaneous analysis, in which we assume that universality is exactly satisfied so that universal parameters occur only once. This feature of the simultaneous analysis, combined with the aforementioned discussion in Sec. I, leads to a significantly improved estimation of the critical points of these systems and the universal quantities including scaling exponents and the Binder ratio $Q^{(0)}$.

A difficulty is that such a simultaneous analysis requires a large amount of accurate Monte Carlo data. Fortunately, some numerical data are already available and were published elsewhere [9-11,22-25]. The data generated by the Cluster Processor [11] are not included and will be published elsewhere. Our Monte Carlo simulations mainly focus on larger system sizes, and were performed on a cluster of 6 PCs with a frequency of $2100 \mathrm{MHz}$.

The outline of this paper is as follows. Section II summarizes the definitions of the aforementioned 11 models, and describes the Monte Carlo techniques involved. Sections III and IV present numerical analyses of the universal ratio $Q$ and of some other quantities, respectively. A discussion is given in Sec. V.

\section{MODELS AND ALGORITHMS}

As mentioned earlier, the present Monte Carlo analyses include eleven Ising-like models. Except the hard-core lattice gas, these models can be represented in terms of a spin-1 Hamiltonian

$$
\begin{aligned}
\mathcal{H} / k_{\mathrm{B}} T= & -K_{\mathrm{NN}} \sum_{\langle\mathrm{NN}\rangle} \sigma_{i} \sigma_{j}-K_{2 \mathrm{~N}} \sum_{(2 \mathrm{~N})} \sigma_{i} \sigma_{j}-K_{3 \mathrm{~N}} \sum_{[3 \mathrm{~N}]} \sigma_{i} \sigma_{j} \\
& -K_{4 \mathrm{~N}} \sum_{\{4 \mathrm{~N}\}} \sigma_{i} \sigma_{j}+D \sum_{i} \sigma_{i}^{2}
\end{aligned}
$$

where the sums $\langle\mathrm{NN}\rangle,(2 \mathrm{~N}),[3 \mathrm{~N}]$, and $\{4 \mathrm{~N}\}$ are, respectively, over nearest-, second-, third-, and fourth-neighbor pairs, and the associated couplings are denoted as $K_{\mathrm{NN}}$, $K_{2 \mathrm{~N}}, K_{3 \mathrm{~N}}$, and $K_{4 \mathrm{~N}}$, respectively. The spins can assume three discrete values $s_{i}=0, \pm 1$, where spins $s=0$ may be referred to as vacancies. The detailed definitions are specified in Table I, where ten models are defined on the simplecubic lattice, and one on the diamond lattice. We define the finite-size parameter $L$ by its relation with the total number $N$ of lattice sites as $N=L^{3}$. Thus, the linear size of the eightsite elementary cell of the diamond lattice is taken to be $L$ 
TABLE II. Number of samples (in $10^{7}$ ) and simulation steps per sample. We use the notation $M \times N$ to indicate that $10^{7} M$ samples have been taken at intervals of $M$ Monte Carlo steps. Smaller system sizes $L$ $<20$ are also included in the analysis. For the lattice gas, simulations steps include one Metropolis sweep for each sample.

\begin{tabular}{|c|c|c|c|c|c|c|c|c|c|}
\hline Model $\backslash L$ & 20 & 22 & 24 & 28 & 32 & 40 & 48 & 64 & 128 \\
\hline 1 & $50 \times 10$ & $50 \times 10$ & $50 \times 10$ & $50 \times 10$ & $40 \times 10$ & $40 \times 10$ & $20 \times 20$ & $10 \times 20$ & $5 \times 40$ \\
\hline 2 & $10 \times 10$ & $10 \times 10$ & $10 \times 10$ & $12 \times 10$ & $20 \times 10$ & $10 \times 10$ & $5 \times 20$ & $5 \times 32$ & $1.8 \times 64$ \\
\hline 3 & $10 \times 10$ & $10 \times 10$ & $10 \times 10$ & $10 \times 10$ & $10 \times 10$ & $10 \times 10$ & $5 \times 20$ & $5 \times 32$ & $2 \times 64$ \\
\hline 4 & $10 \times 10$ & $10 \times 10$ & $10 \times 10$ & $10 \times 10$ & $10 \times 10$ & $10 \times 10$ & $5 \times 20$ & $5 \times 32$ & $2 \times 64$ \\
\hline 5 & $10 \times 10$ & $10 \times 10$ & $10 \times 10$ & $10 \times 10$ & $10 \times 10$ & $10 \times 10$ & $5 \times 20$ & $5 \times 32$ & $2 \times 64$ \\
\hline 6 & $10 \times 10$ & $10 \times 10$ & $10 \times 10$ & $10 \times 10$ & $10 \times 10$ & $10 \times 10$ & $5 \times 20$ & $5 \times 32$ & $2 \times 64$ \\
\hline 7 & $20 \times 20$ & $15 \times 22$ & $17 \times 24$ & $15 \times 28$ & $12 \times 32$ & $8 \times 40$ & $6 \times 48$ & $5 \times 64$ & $3 \times 128$ \\
\hline 8 & $20 \times 20$ & $15 \times 22$ & $12 \times 24$ & $10 \times 28$ & $8 \times 32$ & $6 \times 40$ & $4 \times 48$ & $3 \times 64$ & $2 \times 128$ \\
\hline 9 & $20 \times 20$ & $17 \times 22$ & $12 \times 24$ & $10 \times 28$ & $7 \times 32$ & $6 \times 40$ & $4 \times 48$ & $3 \times 64$ & $1.5 \times 128$ \\
\hline 10 & $128 \times 6$ & $92 \times 6$ & $92 \times 6$ & $92 \times 6$ & $87 \times 8$ & $55 \times 10$ & $22 \times 12$ & $2 \times 16$ & $5.4 \times 25$ \\
\hline 11 & $150 \times 7$ & $20 \times 8$ & $52 \times 10$ & $50 \times 10$ & $30 \times 12$ & $15 \times 14$ & $12 \times 16$ & $15 \times 64$ & $4.4 \times 128$ \\
\hline
\end{tabular}

$=2$. Periodic boundary conditions are applied. The systems sizes were taken in the range $4 \leqslant L \leqslant 128$.

For $D=-\infty$, the vacancies are excluded, so that the model reduces to the spin- $\frac{1}{2}$ model. This applies to the first nine models in Table I. Models 1 and 2 have nearestneighbor interactions $K_{\mathrm{NN}}$ only. Models 3-6 include, in addition, third-neighbor interactions $K_{3 \mathrm{~N}}$. Various ratios are applied: $K_{3 \mathrm{~N}} / K_{\mathrm{NN}}=0.1,0.2,0.3$, and 0.4. Models 7-9 are the equivalent-neighbor models [17-19] of order 2, 3, and 4, respectively. We choose these models because they cover a wide range of amplitudes of the irrelevant field $v$ in Eq. (2). In particular, $v$ is positive for models $1-4$ and negative for models 5-9. This reflects that the critical points of these systems lie on opposite sides of the Ising fixed point in the direction of $v$ on the critical surface. Moreover, the absolute value of $v$ is relatively large for models $1,2,8$, and 9 , and relatively small for models 4,5 , and 7 . This will be shown later in the numerical analysis.

During the Monte Carlo simulations, one can in principle apply the standard form of the Swendsen-Wang or of the Wolff cluster algorithm. However, the efficiency of these methods decreases rapidly as the number of interacting neighbors increases. This difficulty is avoided by an algorithm described in Ref. [9]. Here, we summarize the essential points. During the formation of a cluster, a bond between equal spins coupled with strength $K$ is frozen with probability $p=1-\exp (-2 K)$, or broken with probability $1-p$. Sites connected by frozen bonds belong to the same cluster. The distribution $P(k)=p(1-p)^{k-1}$ expresses the probability that $(k-1)$ subsequent bonds are broken while the $k$ th bond is frozen. The algorithm generates this distribution from a uniformly distributed random number $0<r<1$ as follows:

$$
k=1+[\ln (r) / \ln (1-p)],
$$

where the square brackets denote the integer part. By repeated evaluation of $k$, one may set up a complete list of frozen bonds, and thus a cluster is formed. The efficiency of this procedure is almost independent of the range of the interactions. An example was shown in Ref. [22] by simulating the mean-field Ising model, in which each spin is interacting with every other spin.

We also include a spin-1 model with $D=\ln 2$, which is important to our purposes due to its very small amplitude of $v$ [9]. However, for a general spin-1 model, it is not obvious how cluster algorithms can produce transitions between vacancies and nonvacancies. One can in principle follow a hybrid algorithm in which Metropolis sweeps alternate with cluster steps. As long as the spin-1 model is not close to the tricritical point where the ordered Ising phases meet the phase dominated by vacancies, serious critical slowing down is not expected.

Here, due to the special choice $D=\ln 2(\operatorname{model} 10)$, a full cluster algorithm $[9,11,22]$ becomes possible. First, the spin-1 model is mapped onto a spin- $-\frac{1}{2}$ model with two variables, of which the Hamiltonian is

$$
\mathcal{H} / k_{\mathrm{B}} T=-M_{1} \sum_{\langle i j\rangle}\left(t_{i}+u_{i}\right)\left(t_{j}+u_{j}\right)-M_{2} \sum_{m} t_{m} u_{m},
$$

where two $s=\frac{1}{2}$ spins $t_{i}= \pm 1$ and $u_{i}= \pm 1$ sit on each site $i$ of the simple-cubic lattice. Using the transformations $\sigma_{i}$ $=\left(t_{i}+u_{i}\right) / 2$ and $v_{i}=\left(1+t_{i}\right)\left(1-u_{i}\right) / 4$, it has been shown [9] that the partition function is, up to a constant factor,

$$
Z=\sum_{\sigma_{k}} \exp \left[4 M_{1} \sum_{\langle i j\rangle} \sigma_{i} \sigma_{j}+\left(2 M_{2}-\ln 2\right) \sum_{m} \sigma_{m}^{2}\right]
$$

This is precisely the partition function of the spin- 1 model. The special choice $D=\ln 2$ leads to $M_{2}=0$ so that there are no interactions between variables on the same site. On this basis, the Wolff algorithm is applied to flip the variables $t_{i}$ and/or $u_{i}$. This costs a little price, i.e., two arrays have to be stored in computer memory for the variables $t_{i}$ and $u_{i}$. In the present paper, we improve this algorithm by using one vari- 
TABLE III. Separate fits of the dimensionless ratio $Q$ with $y_{1}$ fixed.

\begin{tabular}{ccccccc}
\hline \hline Model & $K_{c}$ & $Q^{(0)}$ & $q_{1}$ & $q_{2}$ & $b_{1}$ & $q_{1}^{2} / q_{2}$ \\
\hline 1 & $0.36973976(16)$ & $0.62338(8)$ & $0.4906(5)$ & $0.19(1)$ & $0.1150(10)$ & $1.26(7)$ \\
2 & $0.22165452(8)$ & $0.62327(10)$ & $0.885(10)$ & $0.58(5)$ & $0.097(2)$ & $1.35(15)$ \\
3 & $0.18562459(10)$ & $0.62351(10)$ & $0.995(1)$ & $0.80(3)$ & $0.051(2)$ & $1.24(4)$ \\
4 & $0.16073242(15)$ & $0.62364(15)$ & $1.128(13)$ & $1.07(4)$ & $0.0118(20)$ & $1.19(5)$ \\
5 & $0.14230189(10)$ & $0.62355(14)$ & $1.250(13)$ & $1.26(5)$ & $-0.0180(20)$ & $1.24(5)$ \\
6 & $0.12800424(12)$ & $0.62350(16)$ & $1.385(30)$ & $1.85(18)$ & $-0.0480(20)$ & $1.03(15)$ \\
7 & $0.06442225(5)$ & $0.62338(10)$ & $2.854(40)$ & $7.5(2)$ & $-0.0189(20)$ & $1.08(19)$ \\
8 & $0.04303818(3)$ & $0.62324(15)$ & $4.02(30)$ & $15.1(9)$ & $-0.1070(20)$ & $1.07(16)$ \\
9 & $0.03432687(4)$ & $0.62337(26)$ & $4.99(40)$ & $23.2(15)$ & $-0.2123(40)$ & $1.08(23)$ \\
10 & $0.3934222(2)$ & $0.62344(5)$ & $0.6617(8)$ & $0.360(2)$ & $-0.0015(7)$ & $1.21(6)$ \\
11 & $0.0544853(20)$ & $0.62316(20)$ & $0.0593(1)$ & $0.0027(4)$ & $0.212(4)$ & $1.29(12)$ \\
\hline \hline
\end{tabular}

able only. This improvement is based on the equivalence of the variables $t_{i}$ and $u_{i}$. Because of this symmetry, only the sum of $t_{i}$ and $u_{i}$ on the same site needs to be stored. This leads to a cluster algorithm for $D=\ln 2$, which allows flips between nonzero and zero spins.

Another model (model 11) investigated in the present paper is the hard-core lattice gas on the simple-cubic lattice, of which the Hamiltonian is

$$
\mathcal{H} / k_{\mathrm{B}} T=-K \sum_{\langle\mathrm{NN}\rangle} \sigma_{i} \sigma_{j}-\mu \sum_{m} \sigma_{m}
$$

Here, the variable $\sigma_{i}=1,0$ represents the presence and the absence of a particle, respectively. The nearest-neighbor coupling $K \rightarrow-\infty$ implies that no nearest-neighbor sites are allowed to be occupied simultaneously. The chemical potential of the particles is denoted as $\mu$. This lattice gas was Monte Carlo simulated by means of a combination of the Metropolis and a geometric cluster method. This cluster algorithm is based on geometric symmetries, such as the spatial inversion symmetry of the simple-cubic lattice. The full description of this algorithm is given in Refs. [23-25].

As mentioned in Sec. I, the critical behavior of the hardcore lattice gas is expected to belong to the Ising universality class. Surprisingly, significant differences have been reported. The investigations by Yamagata [26,27] yielded critical exponents $\beta / \gamma=0.311(8)$ and $\gamma / v=2.38(2)$, which would imply $y_{h}=2.689(8)$. These results, however, could not be confirmed by later investigations which did reveal a relatively large irrelevant field, but no deviations from the Ising universality class $[23,24]$.

\section{DIMENSIONLESS RATIO $Q$}

For the aforementioned 11 systems, Monte Carlo simulations took place very close to critical points for $L>20$, while ranges of temperaturelike parameters are wider for smaller systems. Table II presents the number of $10^{7}$ of samples taken per system size and the number of simulation sweeps before taking each sample.

During the simulations, the universal ratio $Q$ $=\left\langle m^{2}\right\rangle^{2} /\left\langle m^{4}\right\rangle$ was sampled, where $m$ is the order parameter. For the spin systems (models 1-10) and the lattice gas (model 11), the magnetization density and the staggered particle density assume this role, respectively. Near the critical points, we analyzed $Q$ both separately and simultaneously.

\section{A. Separate analyses}

The finite-size behavior of $Q$ near the critical points is described by Eq. (2). Here, the thermal scaling field $t$ depends on temperaturelike parameters. For the spin- $\frac{1}{2}$ models (models 1-9), these are the spin-spin interactions $K_{\mathrm{NN}}$, $K_{2 \mathrm{~N}}, K_{3 \mathrm{~N}}$, and $K_{4 \mathrm{~N}}$. Since fixed ratios apply between these couplings, it is sufficient to select $K_{\mathrm{NN}}$ as the only temperature parameter $K_{i}$ for the $i$ th model. For the spin-1 model (model 10), both the nearest-neighbor couplings $K_{\mathrm{NN}}$ and the chemical potential $D$ are temperaturelike parameters. In this work, $D$ is fixed at $\ln 2$ so that $K_{i}$ is again represented by $K_{\mathrm{NN}}$. For the hard-core lattice gas (model 11), the chemical potential $\mu$ of the particles assumes this role. For later convenience, near the critical points, we express the dependence of the scaling field $t$ on the physical temperature parameter $K_{i}$ as $t=a_{i}\left(K_{i}-K_{c i}\right)+b_{i}\left(K_{i}-K_{c i}\right)^{2}+\cdots$. The amplitudes of the quantities with the subscript $i$ depend on specific models. On this basis, Eq. (2) becomes

$$
\begin{aligned}
Q= & Q^{(0)}+Q^{(1)} a_{i}\left(K_{i}-K_{c i}\right) L^{y_{t}}+Q^{(2)} a_{i}^{2}\left(K_{i}-K_{c i}\right)^{2} t^{2} L^{2 y_{t}}+Q^{(3)} a_{i}^{3}\left(K_{i}-K_{c i}\right)^{3} t^{3} L^{3 y_{t}} \\
& +Q^{(4)} a_{i}^{4}\left(K_{i}-K_{c i}\right)^{4} t^{4} L^{4 y_{t}}+c_{i}\left(K_{i}-K_{c i}\right)^{2} L^{y_{t}}+b_{1 i} L^{y_{1}}+b_{2 i} L^{y_{2}}+b_{3 i} L^{y_{3}}+\cdots
\end{aligned}
$$


Here, we have written $Q^{(i, 0)}$ as $Q^{(i)}$ for simplicity. The term with the coefficient $c_{i}$ reflects the nonlinear dependence of $t$ on $K_{i}$. The exponents of the correction terms, as obtained in earlier analyses of $Q$ in Refs. [9,11], are $y_{1}=y_{i}=$ $-0.82(3), \quad y_{2}=d-2 y_{h}=-1.963(3)$, and $y_{3}=y_{t}-2 y_{h}=$ $-3.375(3)$. The correction with the exponent $y_{2}$ is due to the field dependence of the analytic part of the free energy. The last term arises from nonlinear dependence of the temperature scaling field on the physical magnetic field. Finitesize scaling also predicts further contributions. For a single model, since both the quantities $Q^{(i)}$ and other parameters such as $a_{i}$ and $K_{c i}$ are unknown, we may simplify Eq. (8) as

$$
\begin{aligned}
Q= & Q_{0}+q_{1 i}\left(K_{i}-K_{c i}\right) L^{y_{t}}+q_{2 i}\left(K_{i}-K_{c i}\right)^{2} L^{2 y_{t}} \\
& +q_{3 i}\left(K_{i}-K_{c i}\right)^{2} L^{3 y_{t}}+q_{4 i}\left(K_{i}-K_{c i}\right)^{2} L^{4 y_{t}} \\
& +c_{i}\left(K_{i}-K_{c i}\right)^{2} L^{y_{t}}+b_{1 i} L^{y_{1}}+b_{2 i} L^{y_{2}}+b_{3 i} L^{y_{3}},
\end{aligned}
$$

where $q_{i j}$ is the product of $Q^{(j)}$ and $a_{i}^{j}$.

According to the least-squares criterion, Eq. (9) was fitted to the Monte Carlo data separately for the aforementioned 11 models. First, we fixed $y_{1}, y_{2}$, and $y_{3}$ at the aforementioned values with the error margins neglected, and $y_{t}$ was taken as 1.587 [3-13]. We applied a cutoff for small system sizes $L$ $<10$ for model $8, L<12$ for model 9 , and $L<8$ for the rest. For the equivalent-neighbor model of order 3 and 4, the Monte Carlo data for small system sizes may be affected by crossover effects due to the proximity of the mean-field fixed point [22]. This is the reason why we applied different cutoffs at small system sizes for models 8 and 9 . The results are shown in Table III. The numerical uncertainties quoted between parenthesis represent one standard deviation. The excellent agreement of the universal quantity $Q^{(0)}$ in the third column confirms that these 11 systems belong to the Ising universality class. Furthermore, according to Eqs. (8) and (9), the quantity $q_{1}^{2} / q_{2}=\left[Q^{(1)}\right]^{2} / Q^{(2)}$ is the same for all Ising-like models. This is confirmed by the last column of Table III, which reveals that the values $q_{1}^{2} / q_{2}$ are consistent with each other within two standard deviations.

The amplitudes of the irrelevant field for these models are shown, up to a constant factor, in the sixth column of Table III. As mentioned in Sec. I, they reflect the positions of the critical points of these systems on the critical surface. For clarity, we start from the Landau-Ginzburg-Wilson description [28] of the Ising model:

$$
-\mathcal{H}(\phi) / k_{\mathrm{B}} T=\int d \mathbf{r}\left[r \phi^{2}(\mathbf{r})+v \phi^{4}(\mathbf{r})+\nabla^{2} \phi(\mathbf{r})+h \phi(\mathbf{r})\right]
$$

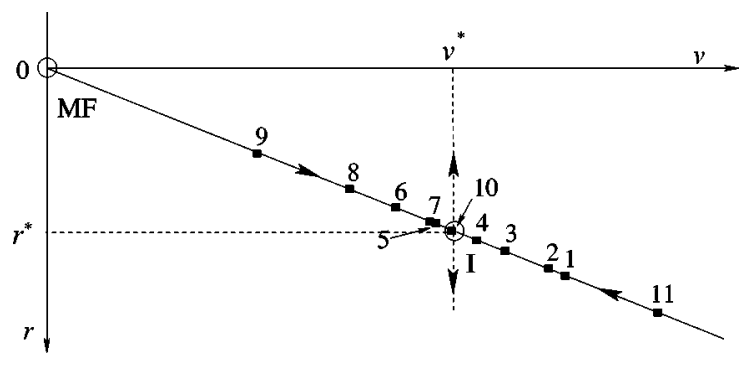

FIG. 1. Schematic illustration of positions of models 1-11 in the parameter space $(r, v)$, where $r$ is a temperaturelike parameter and $u$ reflects the amplitude of the irrelevant field. The mean-field and Ising fixed points, denoted as $\bigcirc$, sit at $(0,0)$ and $\left(r^{*}, v^{*}\right)$, respectively.

where the square-gradient term represents short-range interactions, $r$ and $v$ together determine the temperaturelike and irrelevant parameters, and $h$ is the magnetic field. For spatial dimensionality $d<4$, a renormalization analysis [29] shows that there are two fixed points, i.e., the mean-field $(0,0)$ and the Ising fixed points $\left(r^{*}, v^{*}\right)$ (Fig. 1). The crossover behavior of the Binder ratio $Q^{(0)}$ is displayed by a data collapse in Fig. 10 in Ref. [30]. This provides a scale for the irrelevant Ising field $v$ on the whole range from the Ising to the meanfield fixed point. Using this scale and the value of $b_{1 i}$ for the $i$ th model (Table III), we schematically illustrate the positions of the critical point of the 11 systems in the present work (Fig. 1).

The results in Table III rely on the choice that the irrelevant exponent $y_{i}$ was fixed at -0.82 . As discussed in Sec. I, without such an assumption of the value of $y_{i}$, the accuracy of $Q^{(0)}$ and $y_{i}$ will be very limited. As a test, we left the exponent $y_{1}$ as a free parameter. We find that the uncertainties of $y_{1}$ are then almost as big as the absolute value of $y_{1}$ itself for models with relatively small amplitude $b_{1}$. For the rest, the results in Table III are also affected in the sense that the accuracy decreases significantly, as shown in Table IV.

\section{B. Simultaneous analysis}

On the basis of the universality hypothesis, we analyze the Monte Carlo data of these systems simultaneously. The data were fitted, instead of to Eq. (9), to Eq. (8). As a result, each of the amplitudes $q_{j i}$ is decomposed in a universal factor $Q^{(j)}$ and a nonuniversal factor $a_{i}$. Since the $Q^{(j)}$ are shared by all the systems, the number of unknown parameters decreases significantly, in comparison to the total number in the separate fits. This decomposition also leads to an additional free parameter since numerical data can only determine the product of $Q^{(1)}$ and $a_{i}$, so that one of the pa-

TABLE IV. Some results of separate fits of $Q$ with $y_{1}$ as a free parameter.

\begin{tabular}{cccccc}
\hline \hline Model & 1 & 2 & 8 & 9 & 11 \\
\hline$K_{c}$ & $0.3697399(4)$ & $0.2216545(2)$ & $0.0430382(7)$ & $0.0343268(1)$ & $0.05449(3)$ \\
$Q_{0}$ & $0.6238(7)$ & $0.6231(8)$ & $0.625(1)$ & $0.623(2)$ & $0.625(3)$ \\
$y_{i}$ & $-0.87(6)$ & $-0.800(15)$ & $-0.68(16)$ & $-0.80(2)$ & $-1.0(3)$ \\
\hline \hline
\end{tabular}


TABLE V. Simultaneous fit of the ratio $Q$.

\begin{tabular}{lccccc}
\hline \hline & $Q^{(0)}$ & $Q^{(1)}$ & $Q^{(2)}$ & $Q^{(3)}$ & $Q^{(4)}$ \\
& $0.62342(3)$ & $1($ fixed $)$ & $0.826(6)$ & $-3.32(9)$ & $-9.4(14)$ \\
\hline$y_{i}$ & $K_{c}^{(1)}$ & $K_{c}^{(2)}$ & $K_{c}^{(3)}$ & $K_{c}^{(4)}$ & $K_{c}^{(5)}$ \\
$-0.821(5)$ & $0.36973981(8)$ & $0.22165455(5)$ & $0.18562452(6)$ & $0.16073229(5)$ & $0.14230186(5)$ \\
$K_{c}^{(6)}$ & $K_{c}^{(7)}$ & $K_{c}^{(8)}$ & $K_{c}^{(9)}$ & $K_{c}^{(10)}$ & $K_{c}^{(11)}$ \\
$0.12800417(5)$ & $0.06442222(2)$ & $0.04303821(2)$ & $0.03432687(2)$ & $0.39342225(9)$ & $0.0544876(8)$ \\
$a_{1}$ & $a_{2}$ & $a_{3}$ & $a_{4}$ & $a_{5}$ & $a_{6}$ \\
$0.5203(8)$ & $0.853(1)$ & $0.9930(12)$ & $1.132(2)$ & $1.261(3)$ & $1.390(3)$ \\
$a_{7}$ & $a_{8}$ & $a_{9}$ & $a_{10}$ & $a_{11}$ & $b_{11}$ \\
$2.77(3)$ & $4.031(14)$ & $4.92(3)$ & $0.6603(3)$ & $0.05944(4)$ & $0.114(2)$ \\
$b_{12}$ & $b_{13}$ & $b_{14}$ & $b_{15}$ & $b_{16}$ & $b_{17}$ \\
$0.094(2)$ & $0.052(1)$ & $0.0147(7)$ & $-0.016(1)$ & $-0.046(1)$ & $-0.014(1)$ \\
$b_{18}$ & $b_{19}$ & $b_{110}$ & $b_{111}$ & & \\
$-0.113(2)$ & $-0.219(4)$ & $-0.0012(5)$ & $0.207(4)$ & & \\
\hline \hline
\end{tabular}

rameters $Q^{(j)}(j \neq 0)$ and $a_{i}$ has to be fixed as an arbitrary constant. Here, we simply set $Q^{(1)}=1$. Together with the mechanism discussed in Sec. I, this effect leads to a substantially improved accuracy of the unknown parameters, despite that $y_{i}$ was left as a free parameter. This includes the determination of the critical points, the universal ratio $Q^{(0)}$, the irrelevant exponent $y_{i}$, and the amplitudes $b_{1 i}$. The results are shown in Table $\mathrm{V}$. The amplitude $\left[Q^{(1)}\right]^{2} / Q^{(2)}$ $=1 / 0.826(6)=1.211(9)$ is in good agreement with those in the separate fits (Table III).

\section{OTHER QUANTITIES}

The Monte Carlo simulations also yielded the susceptibility as $\chi=L^{3}\left\langle m^{2}\right\rangle$. Furthermore, we sampled the energy density and its cross products with $\left\langle m^{2}\right\rangle$ and $\left\langle m^{4}\right\rangle$. Thus, we obtained derivative of $Q$, denoted as $Q_{p}$. Analysis of $\chi$ and
$Q_{p}$ yields an estimation of the magnetic and thermal exponents $y_{t}$ and $y_{h}$, respectively.

\section{A. Simultaneous analysis of $\chi$}

According to finite-size scaling, the magnetic susceptibility $\chi$ behaves as

$$
\chi(t, v, L)=\chi(t)+L^{2 y_{h}-d}\left(\frac{\partial h}{\partial H}\right)^{2} \chi\left(L^{y_{t}} t, L^{y_{i}} v, 1\right)
$$

where $x(t)$ arises from the differentiation of the analytical part of the free energy density, $h$ is the magnetic scaling field, and $H$ is the physical magnetic field. The dependence of $h$ on $H$ is not universal and is linearized as $h=\sqrt{w_{i}} H$. Taking into account that $t=a_{i}\left(K_{i}-K_{c i}\right)+b_{i}\left(K_{i}-K_{c i}\right)^{2}$ $+\cdots$, Taylor expansion of Eq. (11) yields

$$
\begin{aligned}
\chi= & x_{i}+s_{i}\left(K_{i}-K_{c i}\right)+L^{2 y_{h}-d_{1}} w_{i}\left[\chi^{(0)}+\chi^{(1)} a_{i}\left(K_{i}-K_{c i}\right) L^{y_{t}}+\chi^{(2)} a_{i}^{2}\left(K_{i}-K_{c i}\right)^{2} L^{2 y_{t}}\right. \\
& \left.\left.+\chi^{(3)} a_{i}^{3}\left(K_{i}-K_{c i}\right)^{3} L^{3 y_{t}}\right]+\chi^{(4)} a_{i}^{4}\left(K_{i}-K_{c i}\right)^{4} L^{4 y_{t}}+b_{i} L^{y_{i}}+c_{i}\left(K_{i}-K_{c i}\right) L^{y_{t}+y_{i}}\right]
\end{aligned}
$$

Here, the $j$ th thermal derivative of $\chi$ at criticality is denoted as $\chi^{(j)}$. For the $i$ th model, the amplitude $a_{i}$ is the same as in Eq. (8). This will be confirmed later. Equation (12) was fitted to the Monte Carlo data, and the result is shown in Table VI. According to similar arguments as mentioned above, there are two extra free parameters in Eq. (12) during the fit. Here, we simply fixed $\chi^{(0)}$ and $\chi^{(1)}$ equal to 1 . The magnetic renormalization exponent is estimated as $y_{h}=2.4816(1)$. This is in excellent agreement with most available results [3-7,8,9-13], and its precision is comparable with the best known value $y_{h}=2.48180(15)$, obtained from a 25 th-order high-temperature expansion [31]. The critical points are consistent with those in Table $\mathrm{V}$. We also calculated the ratio $r_{i}=\left(a_{i}\right)^{(Q)} /\left(a_{i}\right)^{(\chi)}$, where the superscripts $Q$ and $\chi$ represent that the value of $a_{i}$ is taken from Tables V and VI, respectively. The result is shown in Table VII. The consistency of $r_{i}$ among these 11 models confirms that the function of $t$ of $K$ is independent of the type of physical observable.

\section{B. Simultaneous analysis of $Q_{p}$}

During the Monte Carlo simulations, the energy density $e$ was sampled as the nearest-neighbor sum for models 1-10:

$$
e=\left\langle S_{\mathrm{NN}}\right\rangle=\sum_{\langle\mathrm{NN}\rangle}\left\langle\sigma_{i} \sigma_{j}\right\rangle
$$

For the hard-core lattice gas (model 11), the nearest-neighbor 
TABLE VI. Simultaneous fit of the magnetic susceptibility $\chi$.

\begin{tabular}{lccccc}
\hline \hline & $\chi^{(0)}$ & $\chi^{(1)}$ & $\chi^{(2)}$ & $\chi^{(3)}$ & $\chi^{(4)}$ \\
& $1($ fixed $)$ & 1 (fixed) & $0.409(2)$ & $-0.043(1)$ & $-0.075(2)$ \\
\hline$y_{h}$ & $K_{c}^{(1)}$ & $K_{c}^{(2)}$ & $K_{c}^{(3)}$ & $K_{c}^{(4)}$ & $K_{c}^{(5)}$ \\
$2.4816(1)$ & $0.3697398(1)$ & $0.22165457(3)$ & $0.18562459(7)$ & $0.16073233(6)$ & $0.14230183(12)$ \\
$K_{c}^{(6)}$ & $K_{c}^{(7)}$ & $K_{c}^{(8)}$ & $K_{c}^{(9)}$ & $K_{c}^{(10)}$ & $K_{c}^{(11)}$ \\
$0.12800422(5)$ & $0.06442225(3)$ & $0.04303821(2)$ & $0.03432690(3)$ & $0.3934221(1)$ & $0.054487(1)$ \\
$w_{1}$ & $w_{2}$ & $w_{3}$ & $w_{4}$ & $w_{5}$ & $w_{6}$ \\
$1.75(2)$ & $1.55(2)$ & $1.38(2)$ & $1.266(2)$ & $1.187(2)$ & $1.127(2)$ \\
$w_{7}$ & $w_{8}$ & $w_{9}$ & $w_{10}$ & $w_{11}$ & \\
$1.156(2)$ & $0.989(2)$ & $0.875(1)$ & $0.933(1)$ & $0.2192(4)$ & \\
$a_{1}$ & $a_{2}$ & $a_{3}$ & $a_{4}$ & $a_{5}$ & $a_{6}$ \\
$2.00(4)$ & $3.32(2)$ & $3.87(2)$ & $4.45(4)$ & $4.96(6)$ & $5.63(6)$ \\
$a_{7}$ & $a_{8}$ & $a_{9}$ & $a_{10}$ & $a_{11}$ & \\
$10.64(4)$ & $16.16(3)$ & $19.7(2)$ & $2.65(8)$ & $0.2236(2)$ & \\
\hline \hline
\end{tabular}

couplings are infinitely repulsive, and the quantity $e$ is thus defined, instead, as a sum over the next-nearest-neighbor pairs. On this basis, we sampled a quantity $Q_{p}$ which correlates the magnetization distribution with the energy density:

$$
Q_{p}=2 \frac{\left\langle m^{2} S_{\mathrm{NN}}\right\rangle}{\left\langle m^{2}\right\rangle}-\frac{\left\langle m^{4} S_{\mathrm{NN}}\right\rangle}{\left\langle m^{4}\right\rangle}-\left\langle S_{\mathrm{NN}}\right\rangle=\frac{1}{Q} \frac{\partial Q}{\partial t} \frac{\partial t}{\partial K_{\mathrm{NN}}} .
$$

Little additional effort is required for this task since $m^{2}$ and $e$ are already sampled during the Monte Carlo simulations. The quantity $Q_{p}$ has been reported [9-11] to be a good choice in determining the thermal scaling dimension. The reason will be discussed later on the basis of its scaling behavior. For models 3-10, apart from nearest neighbors, the spin-spin interactions occur between second-, third-, and fourth-neighbor pairs. In those cases, the amplitude of $\partial t / \partial K_{\mathrm{NN}}$ is different from the value of $a_{i}$ in the function $t$ $=a_{i}\left(K_{i}-K_{c i}\right)$. According to Eq. (1), near the critical point the quantity $Q_{p}$ behaves as

$$
Q_{p}(t, v, L)=L^{y_{t}} \frac{\partial t}{\partial K_{\mathrm{NN}}} Q_{p}\left(L^{y_{t}} t, L^{y_{i}} v, 1\right) .
$$

Taking into account contributions of $d_{i} L^{y_{2}}$ due to the analytic part of the free energy, we Taylor-expand this equation as

$$
\begin{aligned}
& Q_{p}=L^{y_{t}} p_{i}\left[Q_{p}^{(0)}+Q_{p}^{(1)} a_{i}\left(K_{i}-K_{c i}\right) L^{y_{t}}+Q_{p}^{(2)} a_{i}^{2}\left(K_{i}-K_{c i}\right)^{2} L^{2 y_{t}}+Q_{p}^{(3)} a_{i}^{3}\left(K_{i}-K_{c i}\right)^{3} L^{3 y_{t}}\right. \\
& \left.+Q_{p}^{(4)} a_{i}^{4}\left(K_{i}-K_{c i}\right)^{4} L^{4 y_{t}}+b_{i} L^{y_{i}}+d_{i} L^{y_{2}}+c_{i}\left(K_{i}-K_{c i}\right)\right],
\end{aligned}
$$

where the parameters $Q_{p}^{(j)}$ are universal, and $\partial t / \partial K_{\mathrm{NN}}$ is denoted as $p_{i}$ for the $i$ th model. Compared to the specific heat, the divergence of $Q_{p}$ with respect to the system size $L$ at criticality is much stronger. According to finite-size scaling, the critical specific heat $C$ behaves approximately as $C$ $-C_{0} \propto L^{2 y_{t}-3}$, where $C_{0}$ arises from the analytical part of

TABLE VII. Results for the ratio $r_{i}$.

\begin{tabular}{lccccc}
\hline \hline Model & 1 & 2 & 3 & 4 & 5 \\
$r$ & $0.259(5)$ & $0.258(5)$ & $0.257(6)$ & $0.256(6)$ & $0.254(7)$ \\
\hline 6 & 7 & 8 & 9 & 10 & 11 \\
$0.253(8)$ & $0.259(7)$ & $0.252(8)$ & $0.252(8)$ & $0.259(6)$ & $0.258(6)$ \\
\hline \hline
\end{tabular}

free energy. The exponent $2 y_{t}-3 \simeq 0.174$ is so small that the term with this exponent is normally difficult to separate from the background contribution $C_{0}$ in numerical analyses. Therefore, the quantity $Q_{p}$ serves as a better choice than $C$ to estimate the thermal exponent $y_{t}$. We fitted Eq. (16) to the

TABLE VIII. Result of simultaneous fit of $Q_{p}$.

\begin{tabular}{lccccc}
\hline \hline$L_{\min }$ & $Q_{p}^{(0)}$ & $Q_{p}^{(1)}$ & $Q_{p}^{(2)}$ & $Q_{p}^{(3)}$ & $Q_{p}^{(4)}$ \\
8 & 1 (fixed) & 0.1 (fixed) & $-4.4(5)$ & $-1.2(2)$ & $9.3(23)$ \\
\hline$y_{t}$ & $p_{1}$ & $p_{2}$ & $p_{3}$ & $p_{4}$ & $p_{5}$ \\
$1.58684(14)$ & $0.825(5)$ & $1.355(2)$ & $1.335(2)$ & $1.334(2)$ & $1.343(2)$ \\
$p_{6}$ & $p_{7}$ & $p_{8}$ & $p_{9}$ & $p_{10}$ & $p_{11}$ \\
$1.351(2)$ & $1.422(1)$ & $1.428(1)$ & $1.441(2)$ & $1.058(1)$ & $0.662(1)$ \\
\hline \hline
\end{tabular}


TABLE IX. Summary of results of the scaling exponents and the universal quantity $Q^{(0)}$ for the threedimensional Ising universality class. RG, renormalization of $\phi^{4}$ model; HTE, high-temperature series expansion; MC, Monte Carlo and finite-size scaling; MCRG, Monte Carlo renormalization; CAM, coherentanomaly method.

\begin{tabular}{|c|c|c|c|c|c|c|}
\hline & Year & $y_{t}$ & $y_{h}$ & $y_{i}$ & $Q^{(0)}$ & Method \\
\hline Le Guillou et al. [5] & 1980 & $1.587(4)$ & $2.485(2)$ & $-0.79(3)$ & & RG \\
\hline Nickel and Rehr [32] & 1990 & $1.587(4)$ & $2.4821(4)$ & $-0.83(5)$ & & HTE \\
\hline Nickel [33] & 1991 & 1.587 & 2.4823 & -0.84 & & HTE \\
\hline Baillie et al. [34] & 1992 & $1.602(5)$ & $2.4870(15)$ & -0.8 to -0.85 & & MCRG \\
\hline Landau $[35]$ & 1994 & $1.590(2)$ & $2.482(7)$ & & & MC \\
\hline Kolesik and Suzuki [7] & 1995 & $1.586(4)$ & $2.482(4)$ & & & CAM \\
\hline Blöte et al. [9] & 1995 & $1.587(2)$ & $2.4815(15)$ & $-0.82(6)$ & $0.6233(4)$ & $\mathrm{MC}$ \\
\hline Blöte et al. [11] & 1996 & $1.585(2)$ & $2.4810(10)$ & & & MCRG \\
\hline Guida and Zinn-Justin [14] & 1998 & $1.586(3)$ & $2.483(2)$ & $-0.799(11)$ & & HTE \\
\hline Blöte et al. [10] & 1999 & $1.5865(14)$ & $2.4814(5)$ & $-0.82(3)$ & $0.62358(15)$ & $\mathrm{MC}$ \\
\hline Campostrini et al. [31] & 2002 & $1.5869(4)$ & $2.48180(15)$ & $-0.82(5)$ & & HTE \\
\hline Present & 2003 & $1.5868(3)$ & $2.4816(1)$ & $-0.821(5)$ & $0.623412(25)$ & MC \\
\hline
\end{tabular}

Monte Carlo data, using the critical points as taken from Table V. This is in line with the relatively weak dependence of $Q_{p}$ on the temperaturelike parameters $K$. The results are shown in Table VIII. As possible alternatives, we have included more terms such as $L^{y_{t}+y_{i}}\left(K_{i}-K_{c i}\right)$ within the square brackets of Eq. (16). However, this does not improve the residual $\chi^{2}$ of the fit. The dependence on the cutoff at small system sizes in the fit was also determined. Taking into account these dependences and the uncertainties of the critical points, we estimate the thermal exponent as $y_{t}=1.5868(3)$.

\section{DISCUSSION}

We have performed extensive Monte Carlo simulations of several Ising-like models in three dimensions. These models were selected such that they span a wide range of the irrelevant field, as illustrated in Fig. 1. In order to enable a meaningful test of universality, the models are also chosen according to quite different microscopic Hamiltonians. On the basis of finite-size scaling, we analyze the Monte Carlo data both separately and simultaneously. These systems are confirmed to be within the Ising universality class. Compared to other methods, our simultaneous analyses yield more accurate estimations for the critical points, renormalization exponents, and the Binder cumulant. In particular, we determine the irrelevant exponent as $y_{i}=-0.821(5)$. Tables IX and X show a comparison between some existing results and our estimations.

In order to interpret numerical data correctly, it is necessary to include appropriate corrections to scaling. We find that, normally, a single power-law correction is not sufficient to account for all finite-size corrections. For instance, if one neglects the term $b_{3} L^{y_{3}}$ in Eq. (9), which is decaying relatively fast, one finds a considerable increase of the residual $\chi^{2}$ both in the separate and simultaneous fits. In three dimensions, Monte Carlo simulations are restricted to linear system sizes $L$ in the order of 100 . Even for $L \approx 100$, corrections to scaling are still significant. For instance, we consider the contribution of $b_{1} L^{y_{i}}$ in Eq. (9) for the spin- $\frac{1}{2}$ model on the simple-cubic lattice (model 2$)$. From Table III $\left(b_{1} \approx 0.094\right)$, we find that the term $b_{1} L^{y_{i}}$ contributes about 0.002 to $Q$ for $L=90$. Compared to the accuracy 0.00003 of $Q^{(0)}$ in Table $\mathrm{V}$, this contribution is huge and may not be neglected. Another example of corrections due to the irrelevant field is provided in Ref. [8], where the spontaneous magnetization density $M$ was analyzed as $M(t)=f(t) t^{\beta}$ for the Ising model on simple-cubic lattices with linear sizes up to $L=256$. Here, $t$ is the reduced temperature $t=\left(K-K_{c}\right) / K_{c}$, the exponent $\beta$ is equal to $\left(3-y_{h}\right) / y_{t}$, and $f(t)$ is some function of $t$ that contains the corrections to scaling. It was found that, without including a correction $\sim t^{y_{i} / y_{t}}$ due to the irrelevant field in the function $f(t)$, one cannot successfully describe the numerical data $(0.0005<t<0.26)$, even when $f(t)$ is defined as $f(t)=p_{0}+p_{1} t+p_{2} t^{2}+p_{3} t^{3}$. Another analysis involving the spontaneous magnetization density was recently carried out by García et al. [15]. Remarkably, they claimed that, for $L>90$ and $t>0.004$, corrections to scaling are invisible. They did not comment on the nature of the discrepancy with Ref. [8], and did not provide details about their error esti-

TABLE X. Summary of results for the critical points.

\begin{tabular}{cccc}
\hline \hline Model & $K_{c}$ (present) & $K_{c}$ & $K_{c}$ \\
\hline 1 & $0.36973980(9)$ & $0.36978(4)[21]$ & $0.3697(8)[36]$ \\
2 & $0.22165455(3)$ & $0.221656(10)[9]$ & $0.2216576(22)[35]$ \\
3 & $0.18562452(6)$ & $0.18562466(52)[11]$ & \\
4 & $0.16073229(5)$ & $0.16073235(12)[11]$ & \\
5 & $0.14230186(5)$ & $0.14230187(12)[11]$ & \\
6 & $0.12800417(5)$ & $0.12800393(40)[11]$ & $0.1280039(4)[9]$ \\
7 & $0.06442222(2)$ & $0.0644220(5)[30]$ & $0.06450[17-19]$ \\
8 & $0.04303821(2)$ & $0.0430381(5)[30]$ & $0.0432[17-19]$ \\
9 & $0.03432687(2)$ & $0.03432685(15)[30]$ & \\
10 & $0.39342225(5)$ & $0.3934220(7)[9]$ & \\
11 & $0.0544876(8)$ & $0.05443(7)[24]$ & $0.057136(8)[26]$ \\
\hline \hline
\end{tabular}


mation. Therefore, some doubt concerning the precision of their results $\left[y_{t}=1.600(2)\right.$ and $\left.y_{h}=2.501(5)\right]$ seems justified.

For the spin-1 model and the lattice gas, another quantity of interest is the density of vacancies $\rho_{v}$ at the critical points. Finite-size analysis yields $\rho_{v}=0.400694(1)$ and $0.789516(1)$ for these two models, respectively.

\section{ACKNOWLEDGMENTS}

We are indebted to J.R. Heringa for valuable discussions. This research was supported by the Dutch FOM foundation ("Stichting voor Fundamenteel Onderzoek der Materie") which is financially supported by the NWO ('Nederlandse Organisatie voor Wetenschappelijk Onderzoek').
[1] L. Onsager, Phys. Rev. 65, 117 (1944).

[2] B.M. McCoy and T.T. Wu, The Two-Dimensional Ising Model (Harvard University Press, Cambridge, MA, 1968), and references therein.

[3] J.H. Chen, M.E. Fisher, and B.G. Nickel, Phys. Rev. Lett. 48, 630 (1982).

[4] J. Adler, J. Phys. A 16, 3585 (1983).

[5] J.C. Le Guillou and J. Zinn-Justin, J. Phys. (Paris) 48, 19 (1987).

[6] A.J. Guttmann and I.G. Enting, J. Phys. A 27, 8007 (1994).

[7] M. Kolesik and M. Suzuki, Physica A 215, 138 (1995).

[8] A.L. Talapov and H.W.J. Blöte, J. Phys. A 29, 5727 (1996).

[9] H.W.J. Blöte, E. Luijten, and J.R. Heringa, J. Phys. A 28, 6289 (1995).

[10] H.W.J. Blöte, J.R. Heringa, A. Hoogland, E.W. Meyer, and T.S. Smit, Phys. Rev. Lett. 76, 2613 (1996).

[11] H.W.J. Blöte, L.N. Shchur, and A.L. Talapov, Int. J. Mod. Phys. C 10, 1137 (1999).

[12] M. Hasenbusch, K. Pinn, and S. Vinti, Phys. Rev. B 59, 11471 (1999).

[13] P. Butera and M. Comi, Phys. Rev. B 56, 8212 (1997).

[14] R. Guida and J. Zinn-Justin, J. Phys. A 31, 8103 (1998).

[15] J. García, J.A. Gonzalo, and M.I. Marqués, e-print cond-mat/0211270.

[16] K. Binder, Z. Phys. B: Condens. Matter 43, 119 (1981).

[17] C. Domb, in Phase Transitions and Critical Phenomena, edited by C. Domb and M.S. Green (Academic Press, London, 1974), Vol. 3, p. 357.
[18] N.W. Dalton and C. Domb, Proc. Phys. Soc. London 89, 873 (1966).

[19] C. Domb and N.W. Dalton, Proc. Phys. Soc. London 89, 859 (1966).

[20] J.W. Essam and M.F. Sykes, Physica (Amsterdam) 29, 378 (1963).

[21] M.F. Sykes and D.S. Gaunt, J. Phys. A 6, 643 (1973).

[22] E. Luijten, Interaction Range, Universality and the Upper Critical Dimension (Delft University Press, Delft, 1997), p. 16.

[23] J.R. Heringa and H.W.J. Blöte, Physica A 232, 369 (1996).

[24] J.R. Heringa and H.W.J. Blöte, Physica A 251, 224 (1998).

[25] J.R. Heringa and H.W.J. Blöte, Phys. Rev. E 57, 4976 (1998).

[26] A. Yamagata, Physica A 222, 119 (1995).

[27] A. Yamagata, Physica A 231, 495 (1996).

[28] For an introduction, see, e.g., S.K. Ma, Modern Theory of Critical Phenomena (Addison-Wesley, Redwood, CA, 1976), and references therein.

[29] K.G. Wilson and M.E. Fisher, Phys. Rev. Lett. 28, 240 (1972).

[30] E. Luijten, Phys. Rev. E 59, 4997 (1999).

[31] M. Campostrini, A. Pelissetto, P. Rossi, and E. Vicari, Phys. Rev. E 65, 066127 (2002).

[32] B.G. Nickel and Rehr, J. Stat. Phys. 61, 1 (1990).

[33] B.G. Nickel, Physica A 177, 189 (1991).

[34] C.F. Baillie, R. Gupta, K.A. Hawick, and G.S. Pawley, Phys. Rev. B 45, 10438 (1992).

[35] L.D. Landau, Physica A 205, 41 (1994).

[36] O.G. Mouritsen, J. Phys. C 13, 3909 (1980). 